\title{
Early Prediction of Cognitive Deficit in Very Preterm Infants Using Brain Structural Connectome With Transfer Learning Enhanced Deep Convolutional Neural Networks
}

\author{
Ming Chen 1,2, Hailong Li', Jinghua Wang ${ }^{3}$, Weihong Yuan ${ }^{3,4}$, Mekbib Altaye ${ }^{5,6}$, \\ Nehal A. Parikh ${ }^{1,6}$ and Lili $\mathrm{He}^{1,6 *}$ \\ ${ }^{1}$ The Perinatal Institute, Cincinnati Children's Hospital Medical Center, Cincinnati, OH, United States, ${ }^{2}$ Department \\ of Electronic Engineering and Computing Systems, University of Cincinnati, Cincinnati, OH, United States, ${ }^{3}$ Department \\ of Radiology, University of Cincinnati College of Medicine, Cincinnati, OH, United States, ${ }^{4}$ Department of Radiology, \\ Cincinnati Children's Hospital Medical Center, Cincinnati, $\mathrm{OH}$, United States, ${ }^{5}$ Division of Biostatistics and Epidemiology, \\ Cincinnati Children's Hospital Medical Center, Cincinnati, OH, United States, ${ }^{6}$ Department of Pediatrics, University \\ of Cincinnati College of Medicine, Cincinnati, $\mathrm{OH}$, United States
}

OPEN ACCESS

Edited by:

Hyunjin Park,

Sungkyunkwan University,

South Korea

Reviewed by:

Amy Kuceyeski,

Cornell University, United States

Xi Jiang,

University of Electronic Science and Technology of China, China

${ }^{*}$ Correspondence:

Lili He

lili.he@cchmc.org

Specialty section:

This article was submitted to

Brain Imaging Methods,

a section of the journal

Frontiers in Neuroscience

Received: 17 May 2020

Accepted: 23 July 2020 Published: 18 September 2020

Citation:

Chen M, Li H, Wang J, Yuan W, Altaye M, Parikh NA and He L (2020) Early Prediction of Cognitive Deficit in Very Preterm Infants Using Brain Structural Connectome With Transfer Learning Enhanced Deep

Convolutional Neural Networks.

Front. Neurosci. 14:858

doi: 10.3389/fnins.2020.00858
Up to $40 \%$ of very preterm infants ( $\leq 32$ weeks' gestational age) were identified with a cognitive deficit at 2 years of age. Yet, accurate clinical diagnosis of cognitive deficit cannot be made until early childhood around 3-5 years of age. Recently, brain structural connectome that was constructed by advanced diffusion tensor imaging (DTI) technique has been playing an important role in understanding human cognitive functions. However, available annotated neuroimaging datasets with clinical and outcome information are usually limited and expensive to enlarge in the very preterm infants' studies. These challenges hinder the development of neonatal prognostic tools for early prediction of cognitive deficit in very preterm infants. In this study, we considered the brain structural connectome as a 2D image and applied established deep convolutional neural networks to learn the spatial and topological information of the brain connectome. Furthermore, the transfer learning technique was utilized to mitigate the issue of insufficient training data. As such, we developed a transfer learning enhanced convolutional neural network (TL-CNN) model for early prediction of cognitive assessment at 2 years of age in very preterm infants using brain structural connectome. A total of 110 very preterm infants were enrolled in this work. Brain structural connectome was constructed using DTI images scanned at term-equivalent age. Bayley III cognitive assessments were conducted at 2 years of corrected age. We applied the proposed model to both cognitive deficit classification and continuous cognitive score prediction tasks. The results demonstrated that TL-CNN achieved improved performance compared to multiple peer models. Finally, we identified the brain regions most discriminative to the cognitive deficit. The results suggest that deep learning models may facilitate early prediction of later neurodevelopmental outcomes in very preterm infants at term-equivalent age.

Keywords: convolutional neural network, deep learning, cognitive deficit, transfer learning, structural connectome 


\section{INTRODUCTION}

A high prevalence of long-term cognitive deficit is wellestablished in very preterm infants ( $\leq 32$ weeks' gestational age), with $35-40 \%$ of this population identified with a deficit at 2 years of age (Blencowe et al., 2012; Hamilton et al., 2016). This neurological deficit may affect the infant throughout life, thereby resulting in difficulties in academic skills and building social relationships. Yet, no robust prognostic screening technique is available following neonatal intensive care stay. Typically, an accurate diagnosis of cognitive deficit cannot be made until early childhood around 3-5 years of age. This delayed diagnosis misses the optimal neuroplasticity period of brain development in the first 3 years of life and potentially undermines the effectiveness of early interventions. As such, reproducible approaches that serve as neonatal prognostic tools are needed to fill the gap in our knowledge about the early prediction of cognitive deficit in very preterm infants.

The human brain is a highly interconnected network with coordinated information transfer among individual brain regions (Sporns et al., 2005). Advanced non-invasive neuroimaging MRI techniques have been applied to construct such network representation of the brain, referred to as the brain connectome (Bullmore and Sporns, 2009). Theoretically, a brain connectome is a graph, where vertices represent a set of brain regions of interest (ROIs) and edges represent brain connectivity between ROIs. This brain connectome perspective shifted traditional research that focuses on isolated ROIs toward research on a systematic mechanism incorporating the whole brain. Brain connectome data have very high dimensionality and are intrinsically complex, creating difficulties in designing feature extraction methods and building analysis models. Deep learning has shown great promise in deciphering complex and high dimensional data (e.g., images, signals, and videos) to achieve superior performance in numerous fields, including computer vision, speech recognition, and natural language processing (LeCun et al., 2015). Indeed, numerous studies have applied deep learning approaches to brain connectome for various neurological disorders (Wee et al., 2012; Barkhof et al., 2014; He et al., 2018; Heinsfeld et al., 2018; Li et al., 2018; Sen et al., 2018; Chen et al., 2019).

Brain connectome plays an important role in understanding human cognitive functions (Nagy et al., 2004; Park and Friston, 2013; Petersen and Sporns, 2015). Recent research demonstrated that deep learning models were capable of predicting later cognitive deficits for neonates using brain structural connectome that was constructed by diffusion tensor imaging (DTI) data (Kawahara et al., 2017; Girault et al., 2019). One method to apply deep learning models to brain connectome data is to ignore the topology of the connectome and reshape the adjacency matrix into a vector of features as input (Munsell et al., 2015; Girault et al., 2019). However, the spatial locality (i.e., 2D grid regions of an adjacency matrix) and topological locality information (i.e., rows/columns of an adjacency matrix) in the brain connectome are not utilized, thereby resulting in information loss and potentially compromising the performance of prediction models. Another approach is to apply specialized topological row and column filters on the adjacency matrix of the brain structural connectome to learn the topological relationship between edges (Kawahara et al., 2017). This approach, however, only emphasizes topological locality and discards the spatial locality information (e.g., physically nearby brain ROIs and associated edges) that are intrinsic to any brain ROI parcellation. Since the brain structural connectome is a modular graph that contains clusters of vertices and edges, its adjacency matrix contains hierarchically segregated modules (Park and Friston, 2013). Those topological filters may extract redundant information within connectome modules and may not be efficient for capturing spatial locality. In this work, we consider the adjacency matrix of brain structural connectome as a $2 \mathrm{D}$ image and propose to apply established deep convolutional neural networks (CNNs) to learn the spatial and topological information of the brain connectome.

Although deep CNN models have shown promising results on image classification, those models usually require large datasets for model training. In the studies of very preterm infants, available annotated neuroimaging datasets with clinical and outcome information are usually limited and expensive to enlarge, preventing deep CNN to be directly utilized. Transfer learning (TL) may serve as a potential solution to this challenge. Briefly, TL reuses a pre-trained model designed for one task as a starting point for another related task (Bengio, 2012; Samala et al., 2016, 2018; Shin et al., 2016; Azizi et al., 2017; Kooi et al., 2017; Zheng et al., 2018; Bizzego et al., 2019). Raina et al. (2007) proposed a self-taught learning framework that takes unlabeled images to improve the classification performance of their target classification task. Cheng et al. (2019) transferred image features learned from the early stages of Alzheimer's disease (AD) to improve the prediction of AD diagnosis. Gao et al. (2019) reused pre-trained models based on a large-scale natural image dataset and re-trained a deep learning model for classification of brain activity heatmaps derived from task-based functional MRI data. Recently, we applied the TL technique to a deep neural network (DNN) model for cognitive deficit prediction using brain functional connectome data (He et al., 2018). The DNN model was pre-trained using a large number of brain connectome data in an unsupervised fashion and then fine-tuned with brain connectome data from very preterm infants.

In this study, we proposed a TL-enhanced deep CNN (TL$\mathrm{CNN}$ ) model for early prediction of cognitive deficit at 2 years of age in very preterm infants using brain structural connectome derived from at term DTI data. Specifically, the proposed model contains two modules, a very deep CNN (which was trained with supervision using $\sim 1.2$ million images from the ImageNet database) (Deng et al., 2009) and a "shallow" CNN. With the fixed weighted pre-trained very deep CNN, we only need to train and fine-tune the "shallow" CNN using available very preterm infants' brain connectome data and associated risks of cognitive deficit. For individual very preterm infants, we constructed brain structural connectome using mean fractional anisotropy from their DTI data collected at term-equivalent age. The proposed model is able to evaluate at term whether or not a very preterm infant will have a high risk to develop later cognitive deficits as well as to predict this infant's cognitive assessment [standardized 
Bayley Scales of Infant and Toddler Development, Third Edition (Bayley III) cognitive score] at 2 years of age.

\section{MATERIALS AND METHODS}

\section{Subjects}

The study includes a cohort of 110 very preterm infants, born at 31 weeks gestational age or less from four academic and non-academic centers in Columbus, Ohio, including Nationwide Children's Hospital (NCH), Ohio State University Medical Center, Riverside Hospital, and Mount Carmel St. Ann's Hospital. Infants were enrolled between December 2014 and April 2016. All subjects with any congenital or chromosomal anomalies affecting the central nervous system were excluded. Infants with cyanotic congenital heart disease were also excluded. The study was approved by the Institutional Review Board of $\mathrm{NCH}$. Approval at the other hospitals was obtained through reciprocity agreements that were in place with $\mathrm{NCH}$. Written informed consent was obtained from parents or legal guardians of all infants.

\section{MRI and Cognitive Outcome Acquisition}

Very preterm infants in the cohort were scanned on a $3 \mathrm{~T}$ scanner (Skyra; Siemens Healthcare) at NCH using a 32-channel phased-array head coil. The imaging was performed after the infant was fed and in natural sleep without sedation. Natus Mini Muffs (Natus Medical Inc., Scan Carlos, CA, United States) and InstaPuffy Silicone Earplugs (E.A.R Inc., Boulder, CO, United States) were employed for MRI noise reduction. DTI was acquired with echo-planar imaging using the following parameters (b800/b2000): repetition time $=6972 / 5073 \mathrm{~ms}$; echo time $=88 \mathrm{~ms}$; field of view $=160 \mathrm{~mm} \times 160 \mathrm{~mm}$; in-plane resolution $=2 \mathrm{~mm} \times 2 \mathrm{~mm}$; number of slices $=76$; slice thickness $=1 \mathrm{~mm} ; 64$ non-colinear diffusion-weighted directions; for all images, one volume has no diffusion sensitization; sensitivity encoding factor equates to 2. High-resolution T2weighted anatomical images were acquired with rapid spin-echo sequence: $\mathrm{TR} / \mathrm{TE}=7.3 / 3.4 \mathrm{~ms}$, flip angle $=11^{\circ}$, voxel dimensions $1.0 \mathrm{~mm} \times 1.0 \mathrm{~mm} \times 1.0 \mathrm{~mm}$, scan time $=2: 47 \mathrm{~min}$.

All preterm infants received (Bayley-III) test at 2 years corrected age while blinded to DTI data. The Bayley-III cognitive scores are on a scale of $40-160$, with a mean of 100 and a standard deviation of 15 .

\section{DTI Data Preprocessing}

DTI data were preprocessed using FMRIB's Diffusion Toolbox (in the FMRIB Software Library, FSL, Oxford, United Kingdom) following our previously established pipeline (Yuan et al., 2015). Specifically, head motion and eddy current artifacts were mitigated by aligning all diffusion images to the b0 image via an affine transformation. Diffusion tensor reconstruction and brain fiber tracking were performed in the subject's native space using Diffusion Toolkit/TrackVis (Hess et al., 2006; Wang et al., 2007). Diffusion tensor calculation was based on a linear least-square fitting algorithm, and brain fiber tracking was based on a deterministic tracking algorithm (Wang et al., 2007). The fiber tracking uses an angular threshold of $35^{\circ}$.
The fiber length threshold was set to $5 \mathrm{~mm}$. The obtained fractional anisotropy maps were harmonized using a batcheffect correction algorithm ComBat (Fortin et al., 2017). We use a neonatal Automated Anatomical Labeling (AAL) brain atlas proposed by Shi et al. (2011). For each subject, the high-resolution T2-weighted images were first registered to the b0 image in the subject's native space and then to the neonatal template space to obtain a transformation matrix. Next, the inverse transformation matrix was used to transform the parcellated ROIs from the template space back to the subject's native space (b0).

\section{Whole-Brain Structural Connectome Construction}

A brain connectome is a graph $G=(A, \Omega)$, where vertices $\Omega$ represent a set of ROIs, and $A$ is an adjacency matrix of edges that represent brain connectivity between a pair of ROIs. Ninety ROIs were defined based on a neonatal automated labeling atlas (Shi et al., 2011). The weights of structural connectivity between each pair of ROIs were calculated as the mean FA of all voxels along the WM tract constructed between the two ROIs, resulting in a $90 \times 90$ symmetric adjacency matrix. This was performed using the UCLA Multimodal Connectivity Package (Bassett et al., 2011).

\section{Overview of TL-Enhanced Deep CNN}

The proposed model contains two modules, a very deep CNN (which was trained with supervision using $\sim 1.2$ million images from the ImageNet database) (Deng et al., 2009) and a "shallow" $\mathrm{CNN}$. In Figure 1, we display a two-stage model training procedure in the top two blocks and picture a clinical application in the bottom block, where the proposed model can aid clinicians in the prediction of cognitive deficit using brain structural connectome data. The model training procedure contains two stages: (1) pre-training in the source domain and (2) fine-tuning in the target domain. Specifically, in stage 1, we first pre-trained a deep $\mathrm{CNN}$ to learn the basic transferrable image representation (e.g., edges, shapes, etc.) using a large number of color images and associated image labels (source domain). In stage 2 , we reused the pre-trained model from stage 1 and fine-tuned the model in the target domain with brain structural connectome and associated cognition deficit outcomes.

\section{Pre-training in the Source Domain}

In the source domain, we trained the proposed model to learn transferrable image representation (e.g., edges, shape, and blobs) from diverse objects (e.g., animals, vehicles, human, and natural environments). We defined the task in the source domain as an image classification task. Adjacency matrices of brain connectome are different from those semantic images (dogs, cats, etc.); however, the low-level imaging features (for example straight and curved lines that construct images) are universal to most image analysis tasks. Therefore, the idea behind TL is to treat the pre-trained model as a feature extractor to extract low-level imaging features from the adjacency matrix of a given structural connectome. In this study, we started with 


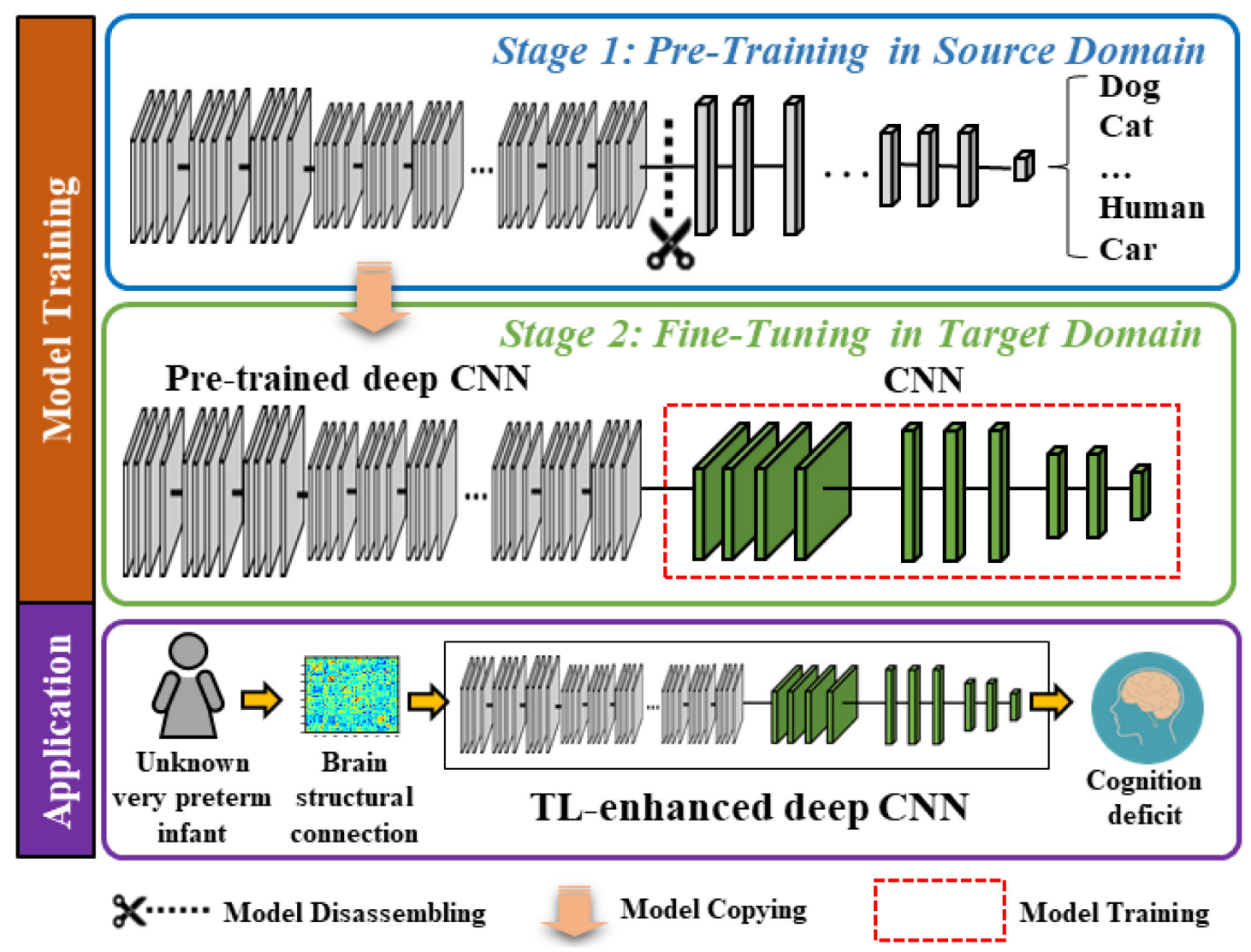

FIGURE 1 | Schematic diagram of the proposed transfer learning-enhanced deep CNN (TL-CNN) model to predict cognitive deficits at 2 years corrected age using brain structural connectome data obtained at term in very preterm infants. The top two blocks demonstrate a two-stage model training procedure, and the bottom block illustrates a potential clinical computer-aided diagnosis application after model training.

the VGG-Nets (Simonyan and Zisserman, 2014) to develop our deep CNN model. VGG-Nets are a set of very deep CNN that were initially proposed by Visual Geometry Group in ImageNet Large Scale Visual Recognition Competition (ILSVRC) 2014. They have been applied to other image analysis applications (Choi et al., 2017; Zhen et al., 2017; Wang et al., 2018). We adopted the architecture of VGG19, one of VGG-Nets models for our study. Briefly, VGG19 is a very deep CNN that consists of 19 trainable layers, including 16 convolutional layers and 3 fully connected (FC) layers designed for classifying 1000 object categories. For each convolutional layer, the VGG19 uses small convolutional filters $(3 \times 3)$ along with a rectified linear unit activation function. We obtained a VGG19 model that was pre-trained using $\sim 1.2$ million color images from the ImageNet database. We then dissembled the model and reserved the weights of the convolutional and pooling layers (Figure 1, blue box).

\section{Fine-Tuning in the Target Domain}

The task in the target domain is to predict the cognitive outcome at 2 years corrected age using brain structural connectome obtained at term-equivalent age. Since the deep CNN in the source domain was pre-trained to recognize transferrable image representation, it would automatically extract image features from the brain structural connectome. The fine-tuning in the target domain is essential to discover discriminative features among generic features and link them to the target task (i.e., cognitive development). We connected a "shallow" CNN (i.e., 2 convolutional layers and 2 FC layers) to the pre-trained fixed weighted deep CNN from the first stage. Finally, an output layer was attached for classification or regression tasks (Figure 1, green box). We used brain structural connectome and follow-up cognitive outcomes to fine-tune the deep CNN model. Given $N$ training samples $\left(\boldsymbol{x}_{1}, \boldsymbol{x}_{2}, \ldots, \boldsymbol{x}_{i}, \ldots, \boldsymbol{x}_{N-1}, \boldsymbol{x}_{N}\right)$ from the target cohort as well as their labels $\left(y_{1}, y_{2}, \ldots, y_{i}, \ldots, y_{N-1}, y_{N}\right)$, where $\boldsymbol{x}_{i}$ is the $i$-th input sample (i.e., brain structural connectome) and $y_{i}$ is the corresponding label, we defined the cross-entropy loss function as:

$$
J(\mathrm{~W}, \boldsymbol{b})=-\frac{1}{N} \sum_{i=1}^{N} y_{i} \log \left(p\left(\boldsymbol{x}_{\boldsymbol{i}}\right)\right)+\left(1-y_{i}\right) \log \left(1-p\left(\boldsymbol{x}_{\boldsymbol{i}}\right)\right)
$$

where $p\left(x_{i}\right)$ is the predicted probability of $x_{i}, W$ is the weight matrix, and $\boldsymbol{b}$ denotes the bias of the model. In addition to the dichotomized prediction (i.e., classification), we also trained our model to perform continuous cognitive score prediction 
(i.e., regression). We applied a linear unit at the end of the model and optimized a weighted mean absolute error (MAE) loss function as follows:

$$
L(\boldsymbol{W}, \boldsymbol{b})=\frac{1}{N} \sum_{i=1}^{N}\left|\left(y_{i}-\hat{y}_{i}(\mathrm{~W}, \boldsymbol{b})\right)\right|
$$

where $\hat{y}_{i}(W, \boldsymbol{b})$ is the output of the linear unit of the model, i.e., the predicted score. Similar to the previous cross-entropy loss function, $\boldsymbol{b}$ represents the bias, and $W$ is the weight of the model. The proposed model was optimized using Adam (Kingma and $\mathrm{Ba}, 2014$ ), a backpropagation gradient descent algorithm. Adam computes adaptive learning rates for weight updating based on the average of recent magnitudes of the gradients, improving computational efficiency. The initial learning rate is set to 0.001 . We applied 50 epochs to train the TL-CNN model. The detailed architecture of the TL-CNN model is elaborated in Supplementary Figure 1.

\section{Alternative Model Comparison Linear/Logistic Regression Model}

In the linear regression model, we applied mean squared error as the loss function to minimize the residual sum of error between the true score and the score predicted by the linear approximation. For the logistic regression (LR) model, we adopted cross-entropy as the cost function. We used L2 regularization as the penalty term, and we grid searched the regularization parameters with empirical values $\left(10^{-3}, 10^{-2}, \ldots, 10^{1}\right)$.

\section{Support Vector Machine}

We tested the support vector machine (SVM) model with three different kernels: linear, polynomial, and radial basis function (RBF), where the SVM with linear kernel achieved the best prediction performance. Specifically, for all SVM models, we used L2 regularization as the penalty. We grid searched the regularization parameters with empirical values $\left(10^{-3}, 10^{-2}, \ldots\right.$, $\left.10^{1}\right)$ and the soft margin parameter $C$ with empirical values $\left(2^{-3}\right.$, $2^{-2}, \ldots, 2^{3}$ ) to optimize the prediction performance. For the polynomial and RBF SVM model, we set the scale gamma kernel coefficient as 1 .

\section{Deep Neural Network}

The DNN model has an input layer, two FC layers with 256 and 64 neurons, and an output layer. The rectifier linear unit as activation function was used in each neuron. We attached a batch normalization layer and a dropout layer after each FC layer. In the output layer, we used a SoftMax classifier for classification and a linear classifier for regression. The DNN was trained in a supervised fashion and tested using the labeled subjects from the target domain.

\section{TL-DNN}

The TL-DNN model has the same structure as the DNN model. Instead of training from scratch, we pre-trained the TLDNN model in an unsupervised fashion using 257 full-termed neonatal subjects from the source domain. Then, we fine-tuned the TL-DNN with supervision using the labeled subjects from the target domain.

\section{Convolutional Neural Network}

The CNN model has two convolutional layers, where each has 256 neurons with a $3 \times 3$ convolutional filter, and two FC layers, where each layer contains 256 and 64 neurons. A rectified linear unit was used as an activation function. A batch normalization and a dropout layer are attached after each FC layer. We applied a SoftMax classifier for the classification task and linear function for the regression task. The architecture design of this model represents a standard "shallow" CNN model without TL strategy. The CNN model was trained and tested using the subjects from the target domain.

\section{Data Augmentation}

The number of very preterm infants in the study cohort is relatively small and imbalanced (i.e., only a small portion of the cohort are at high risk for cognitive deficit). We utilized the synthetic minority over-sampling technique (SMOTE) (Chawla et al., 2002) to balance and augment the training set. Specifically, the training subjects were divided into five bins according to their scores $(<70,70-80,80-90,90-100$, and $>100)$. Given a bin, a sample was randomly chosen. Then, $k$ nearest neighbors for the selected sample were searched. We set $k=5$ in this work. A synthetic sample $x_{\text {syn }}$ is calculated using the randomly selected sample and its associated neighbors $x_{1}, x_{2}, x_{3}, x_{4}, x_{5}, x_{6}$ by: $x_{s y n}=$ $w_{1} x_{1}+w_{2} x_{2}+w_{3} x_{3}+w_{4} x_{4}+w_{5} x_{5}+w_{6} x_{6}$, where $w_{1}, w_{2}, w_{3}$, $w_{4}, w_{5}$, and $w_{6}$ are random numbers and $w_{1}+w_{2}+w_{3}+w_{4}+$ $w_{5}+w_{6}=1$. Similarly, the label $y_{\text {syn }}$ for $x_{\text {syn }}$ was calculated in the same way. The synthetic sample was placed in the given bin. This process is repeated until the number of training subjects reaches 10 times of the original training dataset.

\section{Model Validation}

To evaluate our proposed model, we utilized fivefold crossvalidation for both classification and regression tasks. Specifically, we randomly divided the dataset into five portions. While one portion was used for testing, the remaining four portions were used as training data $(70 \%$ for model training and $30 \%$ for model validation). This process was repeated five times until all portions of the dataset were treated as testing data. We evaluated the performance of risk prediction using accuracy, sensitivity, specificity, and the area under the receiver operating characteristic curve (AUC) across the five iterations. For cognitive score regression, we used Pearson's correlation coefficient, MAE, and standard deviation of absolute error (STD of AE). The fivefold cross-validation experiment was repeated 50 times to reduce the variability and the $95 \%$ confidence interval was reported. All the experiments are performed on a Windows 10 workstation with Intel Xeon Silver $4116 \mathrm{CPU} @ 2.10 \mathrm{GHz}$, 128 GB RAM, and dual GTX 1080ti GPUs.

\section{Most Discriminative Features Detection}

In addition to the prediction of cognitive deficit, we seek to identify which brain regions contributed most to discriminate cognitive deficit. We used gradient-weighted class activation 
mapping (Grad-CAM) to highlight discriminative edges in the brain structural connectome map (Selvaraju et al., 2017). The Grad-CAM produces a coarse localization map highlighting predictive regions in the adjacency matrix by using gradient information of the last convolutional layer of the TL-CNN.

\section{RESULTS}

\section{Subjects}

After excluding the data with large motion artifacts, we had a total of 80 very preterm infants out of 110 subjects in the final analysis. The 80 subjects had a mean (SD) gestational age at birth of 28.0 (2.4) weeks and postmenstrual age at the scan of 40.4 (0.6) weeks. There are 41 (51.3\%) male subjects. The mean (SD) birth weight of the cohort was 1091.5 (385.3) g. We considered the infants with Bayley III cognitive scores $<90$ as a high-risk group (31 subjects) and with Bayley III cognitive scores $\geq 90$ as a low-risk group (49 subjects) to develop later moderate/severe cognitive deficits (Spencer-Smith et al., 2015).

\section{Performance on Risk Stratification of Cognitive Deficits}

We compared the proposed TL-CNN model with LR, linear SVM, and TL-DNN in the identification of very preterm infants at high-risk for moderate/severe cognitive deficits (Table 1). The receiver operating characteristic curves of various machine learning models are displayed in Figure 2. Our proposed TL$\mathrm{CNN}$ model achieved the best prediction performance among the compared models, with $74.5 \%$ on the balanced accuracy, $78.7 \%$ on specificity, $70.2 \%$ on sensitivity, and 0.75 on AUC. The CNN model achieved the lowest balanced accuracy of $67.3 \%$, while DNN had the lowest AUC of 0.59. We also noted that the linear SVM model had better AUC than both DNN and CNN.

Without the TL strategy, the CNN model achieved better accuracy and AUC than DNN. A similar trend was observed on CNN and DNN models with the TL strategy. The TL-DNN achieved $71.6 \%$ on the balanced accuracy, $76.8 \%$ on specificity, $66.4 \%$ on sensitivity, and 0.72 on AUC. The proposed TL-CNN model significantly improved the cognitive deficit prediction over the TL-DNN model by $2.9 \%$ in accuracy $(p=0.005)$ and $3.0 \%$ in AUC $(p=0.008)$. This demonstrated the advantage of treating brain structural connectome as images instead of vectorized weights.
Transfer learning-enhanced models (i.e., TL-DNN and TL$\mathrm{CNN}$ ) had significantly better prediction performance than the models without TL (i.e., DNN and CNN). TL strategy significantly improved prediction accuracy and AUC of CNN by $7.2 \%(p<0.001)$ and $11.6 \%(p<0.001)$. Similarly, TLDNN increased prediction accuracy and AUC of DNN by $2.9 \%$ $(p=0.002)$ and $3.5 \%(p<0.001)$. These results illustrated the effectiveness of the TL approach in deep learning models on the prediction of cognitive deficit.

\section{Performance on the Prediction of Cognitive Scores}

In the regression task, the proposed TL-CNN model had the highest Pearson's correlation coefficient $(r=0.47, p<0.001)$ between the predicted and actual cognitive scores compared to linear regression $(r=0.29, p<0.001)$, support vector regression (SVR) $(r=0.32, p<0.001)$, and TL-DNN $(r=0.37, p<0.001)$ models (Table 2). TL-CNN had the lowest mean STD of AE of 9.5.

\section{Discriminative Brain Structural Connectome}

To reveal which brain regions contributed to the prediction of cognitive deficits, we identified the predictive brain structural connections using the Grad-CAM method (Selvaraju et al., 2017). Table 3 displays the top 15 predictive brain structural connections. We further demonstrated the identified brain connections in a circos plot (Figure 3). The top three discriminative structural connections are located within frontal lobes, limbic lobes, and the subcortical structure. We also plotted those discriminative connections on a brain atlas region using BrainNet Viewer (Xia et al., 2013; Supplementary Figure 2).

\section{DISCUSSION}

Early diagnosis and prediction of cognitive deficit for very preterm infants remain very challenging yet critical for early intervention. In this study, we proposed a TL-CNN model using brain structural connectome at term-equivalent age to predict future cognitive outcomes (i.e., standardized Bayley III cognitive scores). The TL-CNN model achieved promising performance in both risk classification and score regression tasks. For risk prediction of cognitive deficit, TL-CNN achieved a balanced

TABLE 1 | Performance of various machine learning models in utilizing the structural connectome at term-equivalent age to predict cognitive deficits at 2 years corrected age in very preterm infants.



Data in brackets are 95\% confidence intervals. LR, logistic regression; SVM, support vector machine; TL-DNN, transfer learning enhanced deep neural network; TL-CNN, transfer learning enhanced convolutional neural network; AUC, area under the receiver operating characteristic curve. 


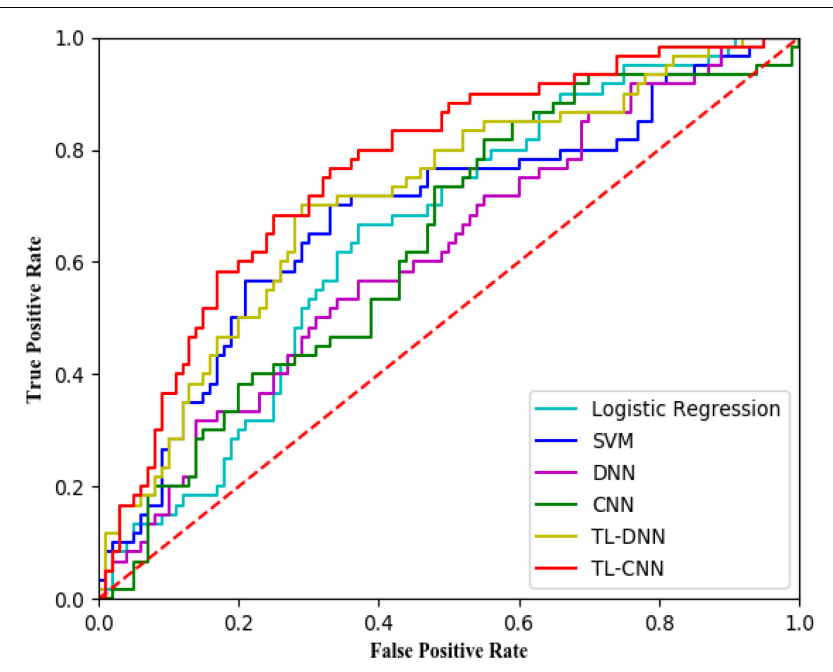

FIGURE 2 | Receiver operating characteristic (ROC) curves of different prediction models using structural brain connectome at term-equivalent age in predicting cognitive deficits at 2 years corrected age in very preterm infants. The proposed TL-CNN model achieved the best area under the ROC curve among compared machine learning models. SVM, support vector machine; DNN, deep neural network; CNN, convolutional neural network; TL-DNN, transfer learning enhanced deep neural network; TL-CNN, transfer learning-enhanced convolutional neural network. accuracy of $74.5 \%$ and an AUC of 0.75 . For regression of cognitive scores, the TL-CNN model had the best Pearson's correlation coefficient among multiple machine learning models. These results demonstrated the feasibility and advantages of a deep learning model that may facilitate the early diagnosis and classification of cognitive deficit for very preterm infants at term-equivalent age.

The proposed TL-CNN model outperformed several peer machine learning models by using both spatial and topological locality information embedded in the adjacency matrix of the brain structural connectome. For those traditional and fully connected neuron-based DNN, the brain connectome is flattened to a vector (He et al., 2018; Chen et al., 2019). This approach discards important spatial and topological locality information from the connectome. By treating the brain structural connectome as 2D images, convolutional filters in $\mathrm{CNN}$ can inherently learn the spatial information. In this study, we adopted a neonatal AAL brain atlas (Shi et al., 2011). The regions in this atlas are numbered through 1-90 and spatially nearby regions have adjacent numbers. In the adjacency matrix of structural connectome, the location of the brain regions follows the original ordering of 1-90; therefore, brain regions near each other in the structural connectome are typically near each other in Euclidean/brain space. In this way, convolutional filters of $\mathrm{CNN}$ are able to learn spatial connectivity information of those "clustered" nearby regions. Meanwhile, those 2D grid convolutional filters move in both row and column directions across the whole adjacency matrix in a single convolutional layer. After a series of consecutive layers, the deep CNN model can integrate the topological locality information gradually. Thus, we believe that applying the deep $\mathrm{CNN}$ model on the adjacency matrix provides unique insight to learn latent spatial and topological locality embedded in the brain structural connectome. The significantly improved prediction performance by the proposed TL-CNN supports the rationale of our study design.

We applied CNN to learn the spatial and topological information of the structural connectome. In this study, we constructed the structural connectome based on a neonatal AAL brain atlas (Shi et al., 2011). The regions in this atlas are numbered through 1-90 and spatially nearby regions have adjacent numbers. Specifically, the neonatal AAL atlas arranged 90 brain regions into the following sections: frontal lobe (region: 1-28, 69-70), occipital lobe (region: 43-54), parietal lobe (region: 61-68), central structures (region: 55-60), and temporal lobe (region: 37-42, 71-90). Therefore, though not strictly speaking, brain regions near each other in the structural connectome are typically near each other in Euclidean/brain space. As CNN's convolutional filters move across rows and columns of the structural connectome adjacency matrix in a moving-windows manner, the model was able to learn topological connectivity information. We tested the prediction performance with five different permuted connectome matrices. The TL-CNN achieved an accuracy of $68.8 \%(95 \% \mathrm{CI}, 66.9,70.7)$, and an AUC of $0.65(95 \% \mathrm{CI}, 0.63,0.67)$, which was slightly lower than the performance of using original structure connectome matrix. This indicates that the order of the ROIs in the structural connectome matrix has an impact on the outcome prediction performance.

Transfer learning technique is essential for studies of very preterm infants using deep learning models. The big data revolution has boosted recent advances in deep learning techniques. Without large training samples, it is very difficult to train a complex deep learning model from scratch. Indeed, the linear SVM demonstrated better performance than deep learning models without the TL strategy in our study. Deep learning models trained with a small number of samples tend to be overfitted. Those relatively simple machine learning models (e.g., SVM) may achieve better performance. Unfortunately, the availability of annotated large brain imaging datasets with clinical and outcome information from very preterm infants is usually very limited, preventing the application of deep learning models in this research domain. The CNN model is a complex network consisting of millions of trainable weights that requires a large amount of data to update the weights when training the model. The TL technique addressed this issue by applying knowledge learned from a large dataset in the source domain to a new target task with limited data to improve performance and model robustness. In the present study, we transferred the knowledge (i.e., optimized weights) from a pre-trained model to the prediction/regression tasks in the target domain and then fine-tuned the model using brain structural connectome to optimize the performance of risk prediction/score regression. The increased performance supports our hypothesis regarding the effectiveness of the TL strategy.

The data balance and augmentation technique also improved the model training. Our dataset was imbalanced with a small number of subjects having low Bayley III cognitive scores. The 
TABLE 2 | Performance of various machine learning models in utilizing the structural connectome at term-equivalent age to predict Bayley-III cognitive scores at 2 years corrected age in very preterm infants.

\begin{tabular}{|c|c|c|c|c|}
\hline Models & $r$ & $p$ & MAE & STD of $A E$ \\
\hline Linear regression & $0.29(0.27,0.31)$ & $<0.0001$ & $20.1(17.6,22.6)$ & $12.0(10.7,13.3)$ \\
\hline SVR & $0.32(0.31,0.34)$ & $<0.0001$ & $18.2(15.1,20.9)$ & $11.4(9.4,13.4)$ \\
\hline TL-DNN & $0.37(0.35,0.39)$ & $<0.0001$ & $22.5(20.0,24.9)$ & $11.2(9.5,13.0)$ \\
\hline TL-CNN & $0.47(0.45,0.49)$ & $<0.0001$ & $16.2(13.8,18.5)$ & $9.5(7.8,11.2)$ \\
\hline
\end{tabular}

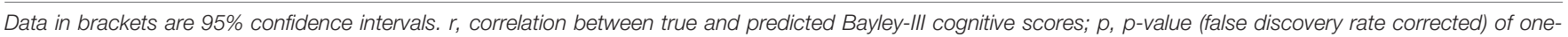

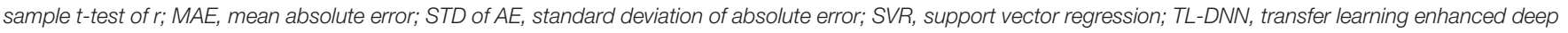
neural network; TL-CNN, transfer learning enhanced convolutional neural network.

TABLE 3 | Top 15 discriminative brain structural connections for prediction of cognitive deficits.

\begin{tabular}{|c|c|c|c|c|}
\hline Brain region $\mathrm{A}$ & Abbreviation & Brain region $\mathbf{B}$ & Abbreviation & $r$ \\
\hline \multicolumn{5}{|l|}{ Top discriminative features } \\
\hline Precentral gyrus left & PreCG-L & Putamen left & PUT-L & 0.39 \\
\hline Superior occipital gyrus left & SOG-L & Superior occipital gyrus right & SOG-R & 0.37 \\
\hline Hippocampus left & HIP-L & Middle occipital gyrus left & MOG-L & 0.34 \\
\hline Postcentral gyrus right & PoCG-R & Putamen right & PUT-R & 0.33 \\
\hline Hippocampus right & HIP-R & Postcentral gyrus right & PoCG-R & 0.33 \\
\hline Hippocampus left & HIP-L & Superior parietal gyrus left & SPG-L & 0.33 \\
\hline Orbitofrontal cortex (superior) left & ORBsup-L & Orbitofrontal cortex (medial) right & ORBmed-R & 0.29 \\
\hline Putamen left & PUT-L & Hippocampus left & HIP-L & 0.28 \\
\hline Postcentral gyrus left & PoCG-L & Putamen left & PUT-L & 0.27 \\
\hline Putamen right & PUT-R & Hippocampus right & HIP-R & -0.25 \\
\hline Postcentral gyrus left & PoCG-L & Hippocampus left & HIP-L & 0.25 \\
\hline Hippocampus right & HIP-R & Thalamus right & THA-R & 0.21 \\
\hline Cuneus left & CUN-L & Precuneus right & PCUN-R & -0.21 \\
\hline Cuneus left & CUN-L & Superior occipital gyrus right & SOG-R & 0.20 \\
\hline Superior frontal gyrus (dorsal) right & SFGdor-R & Hippocampus right & HIP-R & 0.19 \\
\hline
\end{tabular}

r, correlation between brain connectome weights and true Bayley-III cognitive scores.

imbalanced dataset may result in a model that is more likely to predict a high-risk subject into the majority low-risk group. Thus, we applied the data balance and augmentation technique before training any model in this work.

Identification of discriminative brain regions not only improves our understanding of the neurodevelopment of very preterm infants but also enhances the integrity of trained deep learning models. We applied the Grad-CAM method to rank the importance of individual links. Multiple brain regions such as postcentral gyrus, thalamus, and superior occipital gyrus were identified by our TL-CNN model to be predictive to cognitive deficits. These regions were also found to be predictive in our previous study using functional connectome on an independent cohort (He et al., 2018). In addition, postcentral gyrus, thalamus, and superior occipital gyrus were also reported in prior independent studies (Corbetta, 1998; Ouhaz et al., 2018), indicating their association with brain cognitive function. These somatosensory regions are thought to be part of the mirror system, which plays an important role in imitating, understanding, and learning for brain cognitive development (Acharya and Shukla, 2012). Furthermore, the identified most predictive regions have been associated with emotional regulation and memory (limbic lobe) (Catani et al., 2013), visual processing (occipital lobe) (Pöppel et al., 1978), and sensory, visual, and language information processing (parietal lobe) (Wolpert et al., 1998). Additionally, subcortical gray matter regions that play an important role in motion preparation and execution were also ranked highly by the proposed TL-CNN model (Chang et al., 2018).

We further performed a correlation analysis between the top 15 discriminative structural connectome connections and the cognitive outcomes at 2 years corrected age (Table 3 ). Briefly, the majority of brain connectome connections have a positive correlation with the cognitive scores. The increased connectivity strength of these connections would indicate a lower risk of cognitive deficits in very preterm infants at 2 years corrected age. This trend is consistent with our previous study (He and Parikh, 2016). In contrast, two brain connectome connections (Putamen right-Hippocampus right and Cuneus left-Precuneus right) are negatively correlated with cognitive scores, indicating that the increased connectivity strength of these two connections suggests a higher risk of cognitive deficits in very preterm infants at 2 years corrected age. Further investigation is required to unveil the underlying pathological mechanism of these brain connectome connections on brain cognitive functions. 


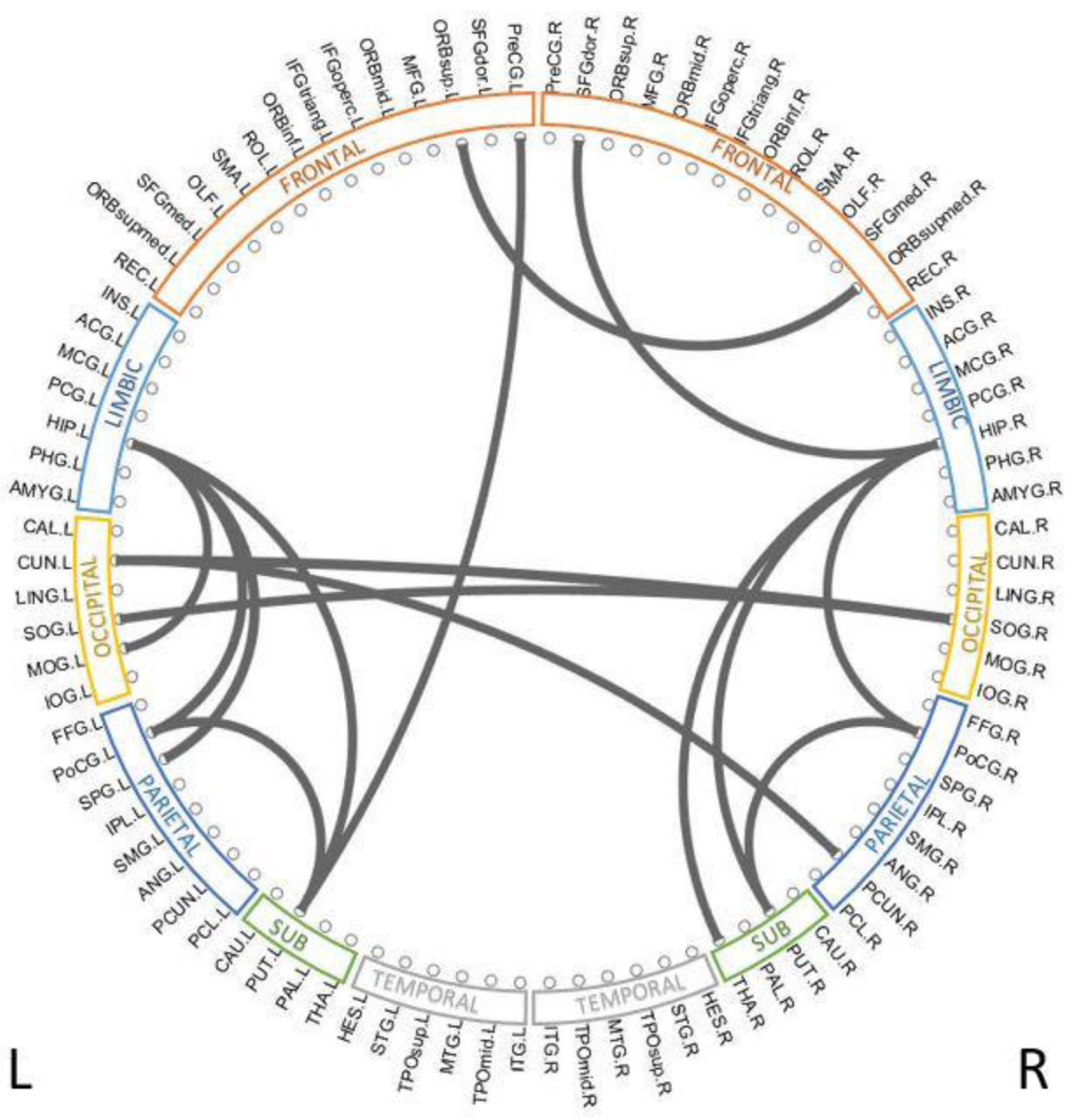

FIGURE 3 | Top 15 discriminative brain structural connections identified by TL-CNN, a circos plot. The top three discriminative structural connections are located within frontal lobes, limbic lobes, and the subcortical structure.

There are several limitations to this study. First, we only internally validated our data in our cohort of very preterm infants. External datasets from independent studies or other research groups are necessary to externally validate the proposed TL-CNN models. Second, we only used brain structural connectome data for the outcome prediction. The integration of brain functional connectome and/or clinical data in our model is likely to improve prediction performance. Third, we constructed brain structural connectome based on an AAL brain atlas without cerebellum regions (Shi et al., 2011). However, the cerebellum regions have been conventionally recognized to have an impact on motor function and recently have been proven to associate with cognitive function (Schmahmann, 2019). The inclusion of the cerebellum regions when we construct the structural connectome may further enhance the prediction performance.

\section{CONCLUSION}

In summary, this study proposed a deep learning model TLCNN for early prediction of cognitive deficit in very preterm infants at 2 years corrected age using brain structural connectome derived from DTI obtained at term-equivalent age. The proposed model achieved improved performance by integrating multiple technique advances, including the convolution of CNN on adjacency matrix, TL strategy, and data balance and augmentation approach. The results suggest that deep learning 
models may facilitate early prediction of later neurodevelopmental outcomes in very preterm infants at term-equivalent age.

\section{DATA AVAILABILITY STATEMENT}

The raw data supporting the conclusions of this article will be made available by the authors, without undue reservation, to any qualified researcher.

\section{ETHICS STATEMENT}

The studies involving human participants were reviewed and approved by the Institutional Review Board Nationwide Children's Hospital. Written informed consent to participate in this study was provided by the participants' legal guardian/next of kin.

\section{AUTHOR CONTRIBUTIONS}

MC, HL, NP, and LH designed the study. MC, HL, JW, and LH conducted the experiments and data analysis. All authors wrote the manuscript.

\section{REFERENCES}

Acharya, S., and Shukla, S. (2012). Mirror neurons: Enigma of the metaphysical modular brain. J. Nat. Sci. Biol. Med. 3, 118-124. doi: 10.4103/0976-9668. 101878

Azizi, S., Mousavi, P., Yan, P., Tahmasebi, A., Kwak, J. T., Xu, S., et al. (2017). Transfer learning from RF to B-mode temporal enhanced ultrasound features for prostate cancer detection. Int. J. Comput. Assist. Radiol. Surg. 12, 1111-1121. doi: $10.1007 /$ s11548-017-1573-x

Barkhof, F., Haller, S., and Rombouts, S. A. (2014). Resting-state functional MR imaging: a new window to the brain. Radiology 272, 29-49. doi: 10.1148/radiol. 14132388

Bassett, D. S., Brown, J. A., Deshpande, V., Carlson, J. M., and Grafton, S. T. (2011). Conserved and variable architecture of human white matter connectivity. Neuroimage 54, 1262-1279. doi: 10.1016/j.neuroimage.2010.09.006

Bengio, Y. (2012). "Deep Learning of Representations for Unsupervised and Transfer Learning," in Paper presented at the Proceedings of ICML Workshop on Unsupervised and Transfer Learning, (Berlin: Springer).

Bizzego, A., Bussola, N., Chierici, M., Maggio, V., Francescatto, M., Cima, L., et al. (2019). Evaluating reproducibility of AI algorithms in digital pathology with DAPPER. PLoS Comput. Biol. 15:e1006269. doi: 10.1371/journal.pcbi.100 6269

Blencowe, H., Cousens, S., Oestergaard, M. Z., Chou, D., Moller, A. B., Narwal, R., et al. (2012). National, regional, and worldwide estimates of preterm birth rates in the year 2010 with time trends since 1990 for selected countries: a systematic analysis and implications. Lancet 379, 2162-2172. doi: 10.1016/S0140-6736(12) 60820-4

Bullmore, E., and Sporns, O. (2009). Complex brain networks: graph theoretical analysis of structural and functional systems. Nat. Rev. Neurosci. 10, 186-198. doi: $10.1038 / \mathrm{nrn} 2575$

Catani, M., Dell'acqua, F., and Thiebaut de Schotten, M. (2013). A revised limbic system model for memory, emotion and behaviour. Neurosci. Biobehav. Rev. 37, 1724-1737. doi: 10.1016/j.neubiorev.2013.07.001

Chang, D. H. F., Ban, H., Ikegaya, Y., Fujita, I., and Troje, N. F. (2018). Cortical and subcortical responses to biological motion. Neuroimage 174, 87-96. doi: 10.1016/j.neuroimage.2018.03.013

\section{FUNDING}

This study was supported by the National Institutes of Health grants R21-HD094085, R01-NS094200, and R01NS096037 and a Trustee grant from Cincinnati Children's Hospital Medical Center.

\section{ACKNOWLEDGMENTS}

We sincerely thank Jennifer Notestine, RN, Valerie Marburger, NNP, Julie Gutentag, RN, and Patty Luzader, RN, for serving as the study coordinators and Mark Smith, MS, for serving as the study MR tech. We are grateful to the families and neonatal intensive care unit and clinic staff that made this study possible.

\section{SUPPLEMENTARY MATERIAL}

The Supplementary Material for this article can be found online at: https://www.frontiersin.org/articles/10.3389/fnins. 2020.00858/full\#supplementary-material

Chawla, N. V., Bowyer, K. W., Hall, L. O., and Kegelmeyer, W. P. (2002). SMOTE: Synthetic minority over-sampling technique. J. Ar. Intell. Res. 16, 321-357. doi: 10.1613/jair.953

Chen, M., Li, H., Wang, J., Dillman, J. R., Parikh, N. A., and He, L. (2019). A Multichannel Deep Neural Network Model Analyzing Multiscale Functional Brain Connectome Data for Attention Deficit Hyperactivity Disorder Detection. Radiol. Ar. Intell. 2:e190012. doi: 10.1148/ryai.2019190012

Cheng, B., Liu, M. X., Zhang, D. Q., Shen, D. G., and Alzheimer's Disease Neuroimaging Initiative (2019). Robust multi-label transfer feature learning for early diagnosis of Alzheimer's disease. Brain Imaging Behav. 13, 138-153. doi: 10.1007/s11682-018-9846-8

Choi, J. Y., Yoo, T. K., Seo, J. G., Kwak, J., Um, T. T., and Rim, T. H. (2017). Multicategorical deep learning neural network to classify retinal images: A pilot study employing small database. PLoS One 12:e0187336. doi: 10.1371/journal.pone. 0187336

Corbetta, M. (1998). Frontoparietal cortical networks for directing attention and the eye to visual locations: Identical, independent, or overlapping neural systems? Proc. Nat. Acad. Sci. Unit. Stat. Am. 95, 831-838. doi: 10.1073/pnas. 95.3.831

Deng, J., Dong, W., Socher, R., Li, L. J., Li, K., and Li, F. F. (2009). “ImageNet: A Large-Scale Hierarchical Image Database," in Cvpr: 2009 Ieee Conference on Computer Vision and Pattern Recognition, (New Jersey: IEEE).

Fortin, J. P., Parker, D., Tunc, B., Watanabe, T., Elliott, M. A., Ruparel, K., et al. (2017). Harmonization of multi-site diffusion tensor imaging data. Neuroimage 161, 149-170. doi: 10.1016/j.neuroimage.2017.08.047

Gao, Y. F., Zhang, Y. M., Wang, H. L., Guo, X. J., and Zhang, J. C. (2019). Decoding Behavior Tasks From Brain Activity Using Deep Transfer Learning. IEEE Access 7, 43222-43232. doi: 10.1109/Access.2019.2907040

Girault, J. B., Munsell, B. C., Puechmaille, D., Goldman, B. D., Prieto, J. C., Styner, M., et al. (2019). White matter connectomes at birth accurately predict cognitive abilities at age 2. NeuroImage 192, 145-155. doi: 10.1016/j.neuroimage.2019. 02.060

Gupta, A., Ayhan, M. S., and Maida, A. S. (2013). Natural image bases to represent neuroimaging data. Atlanta, GA: JMLR.org.

Hamilton, B. E., Martin, J. A., and Osterman, M. J. (2016). Births: Preliminary Data for 2015. Natl. Vital. Stat. Rep. 65, 1-15. 
He, L., Li, H., Holland, S. K., Yuan, W., Altaye, M., and Parikh, N. A. (2018). Early prediction of cognitive deficits in very preterm infants using functional connectome data in an artificial neural network framework. Neuroim. Clin. 18, 290-297. doi: 10.1016/j.nicl.2018.01.032

He, L., and Parikh, N. A. (2016). Brain functional network connectivity development in very preterm infants: The first six months. Early Hum. Dev. 98, 29-35. doi: 10.1016/j.earlhumdev.2016.06.002

Heinsfeld, A. S., Franco, A. R., Craddock, R. C., Buchweitz, A., and Meneguzzi, F. (2018). Identification of autism spectrum disorder using deep learning and the ABIDE dataset. NeuroImag. Clin. 17, 16-23. doi: 10.1016/j.nicl.2017.08.017

Hess, C. P., Mukherjee, P., Han, E. T., Xu, D., and Vigneron, D. B. (2006). Q-ball reconstruction of multimodal fiber orientations using the spherical harmonic basis. Magnet. Reson. Med. 56, 104-117. doi: 10.1002/mrm.20931

Kawahara, J., Brown, C. J., Miller, S. P., Booth, B. G., Chau, V., Grunau, R. E., et al. (2017). BrainNetCNN: Convolutional neural networks for brain networks; towards predicting neurodevelopment. Neuroimage 146, 1038-1049. doi: 10. 1016/j.neuroimage.2016.09.046

Kingma, D. P., and Ba, J. J. (2014). Adam: A method for stochastic optimization. arXiv

Kooi, T., van Ginneken, B., Karssemeijer, N., and den Heeten, A. (2017). Discriminating solitary cysts from soft tissue lesions in mammography using a pretrained deep convolutional neural network. Med. Phys. 44, 1017-1027. doi: $10.1002 / \mathrm{mp} .12110$

LeCun, Y., Bengio, Y., and Hinton, G. (2015). Deep learning. Nature 521, 436-444. doi: $10.1038 /$ nature 14539

Li, H. L., Parikh, N. A., and He, L. L. (2018). A Novel Transfer Learning Approach to Enhance Deep Neural Network Classification of Brain Functional Connectomes. Front. Neurosci. 12:491. doi: 10.3389/fnins.2018.00491

Munsell, B. C., Wee, C. Y., Keller, S. S., Weber, B., Elger, C., da Silva, L. A. T., et al. (2015). Evaluation of machine learning algorithms for treatment outcome prediction in patients with epilepsy based on structural connectome data. Neuroimage 118, 219-230. doi: 10.1016/j.neuroimage.2015.06.008

Nagy, Z., Westerberg, H., and Klingberg, T. (2004). Maturation of white matter is associated with the development of cognitive functions during childhood. J. Cogn. Neurosci. 16, 1227-1233. doi: 10.1162/0898929041920441

Ouhaz, Z., Fleming, H., and Mitchell, A. S. (2018). Cognitive Functions and Neurodevelopmental Disorders Involving the Prefrontal Cortex and Mediodorsal Thalamus. Front. Neurosci. 12:3310. doi: 10.3389/fnins.2018. 00033

Park, H.-J., and Friston, K. J. S. (2013). Structural and functional brain networks: from connections to cognition. Science 342:1238411. doi: 10.1126/science. 1238411

Petersen, S. E., and Sporns, O. J. N. (2015). Brain networks and cognitive architectures. Neuron 88, 207-219. doi: 10.1016/j.neuron.2015.09.027

Pöppel, E., Brinkmann, R., von Cramon, D., and Singer, W. (1978). Association and dissociation of visual functions in a case of bilateral occipital lobe infarction. Arch. Psyc. Nervenkrankheiten 225, 1-21. doi: 10.1007/bf00367348

Raina, R., Battle, A., Lee, H., Packer, B., and Ng, A. Y. (2007). Self-taught learning: transfer learning from unlabeled data. Oregon, USA: Association for Computing Machinery.

Samala, R. K., Chan, H. P., Hadjiiski, L., Helvie, M. A., Wei, J., and Cha, K. (2016). Mass detection in digital breast tomosynthesis: Deep convolutional neural network with transfer learning from mammography. Med. Phys. 43:6654. doi: $10.1118 / 1.4967345$

Samala, R. K., Chan, H. P., Hadjiiski, L. M., Helvie, M. A., Richter, C., and Cha, K. (2018). Evolutionary pruning of transfer learned deep convolutional neural network for breast cancer diagnosis in digital breast tomosynthesis. Phys. Med. Biol. 63:095005. doi: 10.1088/1361-6560/aabb5b

Schmahmann, J. D. (2019). The cerebellum and cognition. Neurosci. Lett. 688, 62-75. doi: 10.1016/j.neulet.2018.07.005
Selvaraju, R. R., Cogswell, M., Das, A., Vedantam, R., Parikh, D., and Batra, D. (2017). "Grad-cam: Visual explanations from deep networks via gradient-based localization," in Paper presented at the Proceedings of the IEEE international conference on computer vision, (New Jersey: IEEE).

Sen, B., Borle, N. C., Greiner, R., and Brown, M. R. G. (2018). A general prediction model for the detection of ADHD and Autism using structural and functional MRI. PLoS One 13:e0194856. doi: 10.1371/journal.pone.0194856

Shi, F., Yap, P. T., Wu, G. R., Jia, H. J., Gilmore, J. H., Lin, W. L., et al. (2011). Infant Brain Atlases from Neonates to 1-and 2-Year-Olds. PLoS One 6:e1874610. doi: 10.1371/journal.pone.0018746

Shin, H. C., Roth, H. R., Gao, M., Lu, L., Xu, Z., Nogues, I., et al. (2016). Deep Convolutional Neural Networks for Computer-Aided Detection: CNN Architectures, Dataset Characteristics and Transfer Learning. IEEE Trans. Med, Imag. 35, 1285-1298. doi: 10.1109/TMI.2016.2528162

Simonyan, K., and Zisserman, A. J. (2014). Very deep convolutional networks for large-scale image recognition. arXiv

Spencer-Smith, M. M., Spittle, A. J., Lee, K. J., Doyle, L. W., and Anderson, P. J. (2015). Bayley-III Cognitive and Language Scales in Preterm Children. Pediatrics 135, e1258-e1265. doi: 10.1542/peds.2014-3039

Sporns, O., Tononi, G., and Kötter, R. (2005). The human connectome: a structural description of the human brain. PLoS comput. Biol. 1:e42. doi: 10.1371/journal. pcbi.0010042

Wang, R., Benner, T., Sorensen, A. G., and Wedeen, V. J. (2007). Diffusion toolkit: a software package for diffusion imaging data processing and tractography. Proc. Intl. Soc. Mag. Reson Med. 15:3720.

Wang, Y., Wang, C., and Zhang, H. (2018). Ship Classification in High-Resolution SAR Images Using Deep Learning of Small Datasets. Sensors 18:2929. doi: $10.3390 / \mathrm{s} 18092929$

Wee, C. Y., Yap, P. T., Zhang, D., Denny, K., Browndyke, J. N., Potter, G. G., et al. (2012). Identification of MCI individuals using structural and functional connectivity networks. Neuroimage 59, 2045-2056. doi: 10.1016/j.neuroimage. 2011.10.015

Wolpert, D. M., Goodbody, S. J., and Husain, M. (1998). Maintaining internal representations: the role of the human superior parietal lobe. Nat. Neurosci. 1, 529-533. doi: $10.1038 / 2245$

Xia, M. R., Wang, J. H., and He, Y. (2013). BrainNet Viewer: A Network Visualization Tool for Human Brain Connectomics. PLoS One 8:e6891010. doi: 10.1371/journal.pone.0068910

Yuan, W. H., Wade, S. L., and Babcock, L. (2015). Structural Connectivity Abnormality in Children with Acute Mild Traumatic Brain Injury using Graph Theoretical Analysis. Hum. Brain Map. 36, 779-792. doi: 10.1002/hbm.22664

Zhen, X., Chen, J., Zhong, Z., Hrycushko, B., Zhou, L., Jiang, S., et al. (2017). Deep convolutional neural network with transfer learning for rectum toxicity prediction in cervical cancer radiotherapy: a feasibility study. Phys. Med. Biol. 62, 8246-8263. doi: 10.1088/1361-6560/aa8d09

Zheng, J., Miao, S., Jane Wang, Z., and Liao, R. (2018). Pairwise domain adaptation module for CNN-based 2-D/3-D registration. J. Med. Imaging 5:021204. doi: 10.1117/1.JMI.5.2.021204

Conflict of Interest: The authors declare that the research was conducted in the absence of any commercial or financial relationships that could be construed as a potential conflict of interest.

Copyright (๑) 2020 Chen, Li, Wang, Yuan, Altaye, Parikh and He. This is an openaccess article distributed under the terms of the Creative Commons Attribution License (CC BY). The use, distribution or reproduction in other forums is permitted, provided the original author(s) and the copyright owner(s) are credited and that the original publication in this journal is cited, in accordance with accepted academic practice. No use, distribution or reproduction is permitted which does not comply with these terms. 